\title{
Microphase Separation of a PS-b-PFS Block Copolymer via Solvent Annealing: Effect of Solvent, Substrate, and Exposure Time on Morphology
}

\author{
Colm T. O'Mahony, ${ }^{1,2}$ Dipu Borah, ${ }^{1,2}$ and Michael A. Morris ${ }^{1,2,3}$ \\ ${ }^{1}$ Materials Chemistry Section, Department of Chemistry, University College Cork, Cork, Ireland \\ ${ }^{2}$ Centre for Research on Adaptive Nanostructures and Nanodevices (CRANN), Trinity College Dublin, Dublin, Ireland \\ ${ }^{3}$ Micro-Nano Centre, Tyndall National Institute, Lee Maltings, Cork, Ireland \\ Correspondence should be addressed to Dipu Borah; d.borah@ucc.ie
}

Received 30 December 2014; Accepted 18 February 2015

Academic Editor: Frederik Wurm

Copyright ( 2015 Colm T. O’Mahony et al. This is an open access article distributed under the Creative Commons Attribution License, which permits unrestricted use, distribution, and reproduction in any medium, provided the original work is properly cited.

Block copolymer (BCP) lithography makes use of the microphase separation properties of BCPs to pattern ordered nanoscale features over large areas. This work presents the microphase separation of an asymmetric polystyrene-block-poly(ferrocenyl dimethylsilane) (PS- $b$-PFS) BCP that allows ordered arrays of nanostructures to be formed by spin casting PS- $b$-PFS on substrates and subsequent solvent annealing. The effects of the solvent annealing conditions on self-assembly and structural stability are discussed.

\section{Introduction}

Within the microelectronics industry, the continuing drive towards ever-smaller device dimension is leading to a revaluation of the current top-down fabrication techniques such as photolithography [1]. It is clear that current methodology, at $193 \mathrm{~nm}$ wavelength, is not consistent with extensibility to ultrasmall feature sizes [2]. Advanced lithographic techniques, currently being explored as potential replacements for conventional photolithography, include extreme UV (EUV) lithography, X-ray lithography, and electron-beam writing but they are not without problems $[2,3]$. While X-ray and e-beam lithographic processes do not face wavelength limitations both processes are impractical in a production setting, due to both high capital costs and long production times $[2,3]$. EUV is the front runner of these techniques. However, light at these wavelengths is absorbed rather than transmitted by conventional lenses and an extensive and expensive overhaul of the current lithographic setup would be required [3]. With these problems in mind, the next decade will see fundamental changes the microelectronics industry as researchers begin focusing an ever increasing amount of resources into the development of "bottom-up" techniques that may not only solve the problems of size limitations but also help reduce cost.

Block copolymers (BCPs) have gained increasing attention as templates and scaffolds for the fabrication of highdensity arrays of nanostructures because of the size and tenability of the microdomains, the ease of processing on flat and patterned surfaces without introducing disruptive technologies, and the ability to manipulate their functionality [3-6]. Various approaches to control the orientation of the microdomains of BCPs include the use of thermal gradients [7], solvent fields [8-11], electric fields [12], chemically patterned substrates [13], graphoepitaxy [14], and controlled interfacial interactions [15] have been examined. The BCP patterns can be transferred to the substrate through a polymer selective etch, the height of the transferred features is determined principally by the etch contrast of the blocks. For high etch selectivity it would be advantageous to use a $\mathrm{BCP}$ where one block contains an inorganic element, like $\mathrm{Si}$ [14] or $\mathrm{Fe}$ [16-18], or in the case of polystyrene-block-poly(ferrocenyl dimethylsilane) (PS- $b$-PFS) both. 
Here, we report that by annealing PS- $b$-PFS thin films by solvent, arrays of cylindrical microdomains with a high degree of parallel order can be achieved with little change of the interdomain distance. Also reported is the effect that annealing time plays in the structural stability of the microphase separation on a variety of different substrates. The results described here are obtained by very simple fabrication steps that are cost-effective and compatible with current industrial processes.

\section{Materials and Methods}

2.1. Materials. An asymmetric polystyrene-block-poly(ferrocenyl dimethylsilane) (PS- $b$-PFS) BCP with a molecular weight of 90,000 $\mathrm{g} \mathrm{mol}^{-1}$ (PS: $M_{n}=60,000 \mathrm{~g} \mathrm{~mol}^{-1}$, PFS: $M_{n}=30,000 \mathrm{~g} \mathrm{~mol}^{-1}, M_{w} / M_{n}=1.2, \Phi_{\mathrm{PS}}=0.67$, and $\Phi_{\mathrm{PFS}}=0.33$, where $M_{n}, M_{w}, \Phi_{\mathrm{PS}}$, and $\Phi_{\mathrm{PFS}}$ are the number average molecular weight, weight average molecular weight, volume fraction of PS, and volume fraction of PFS, resp.), a hydroxyl-terminated random polystyrene-randompoly(methylmethacrylate) (HO-PS- $r$-PMMA) copolymer $\left(M_{n}\right.$ and $M_{w} / M_{n}$ were $12,400 \mathrm{~g} \mathrm{~mol}^{-1}$ and 1.25 , resp.), a polystyrene (PS) homopolymer $\left(M_{n}: 38,000 \mathrm{~g} \mathrm{~mol}^{-1}\right.$ and $M_{w} / M_{n}=1.09$ ), and a poly(ferrocenyldimethylsilane) (PFS) homopolymer $\left(M_{n}: 7,000 \mathrm{~g} \mathrm{~mol}^{-1}\right.$ and $\left.M_{w} / M_{n}=1.3\right)$ were purchased from Polymer Source, Inc., Canada, and were used without further purification. Toluene, tetrahydrofuran (THF), acetone, hexane, heptane, cyclohexane, isopropanol (IPA), ethanol, sulphuric acid, and hydrogen peroxide were of analytical grade purchased from Sigma-Aldrich and used as received. The substrates used were $\langle 100\rangle$ orientated silicon (Si), silicon nitride $\left(\mathrm{Si}_{3} \mathrm{~N}_{4}\right)$, silicon oxynitride $\left(\mathrm{SiO}_{x} \mathrm{~N}_{y}\right)$, germanium $(\mathrm{Ge})$, tungsten $(\mathrm{W})$, and grapheme and were generously provided by Intel, Ireland. Deionized (DI) water was used wherever necessary.

2.2. Substrate Cleaning/Activation. Substrates were cleaned either by sonication in toluene or in a piranha solution. Prior to cleaning, substrates were degreased by ultrasonication in acetone and IPA solutions for 5 min each, dried in flowing $\mathrm{N}_{2}$ gas, and baked for $2 \mathrm{~min}$ at $120^{\circ} \mathrm{C}$ in an ambient atmosphere to remove any residual IPA. Piranha activation was carried out in a piranha solution $\left(1: 3 \mathrm{v} / \mathrm{v} 30 \% \mathrm{H}_{2} \mathrm{O}_{2}: \mathrm{H}_{2} \mathrm{SO}_{4}\right)$ (may cause explosion in contact with organic material) at $90^{\circ} \mathrm{C}$ for $60 \mathrm{~min}$, rinsed with DI water several times, acetone, ethanol, and dried under $\mathrm{N}_{2}$ flow. Piranha treatment removes any organic contaminant and creates hydroxyl groups on the $\mathrm{Si}$ substrates.

2.3. Polymer Brush Immobilization on Si Substrate. A HOPS- $r$-PMMA brush solution of 1.0 wt.\% in toluene was spincoated (P6700 Series Spin-coater, Speciality Coating Systems, Inc., USA) onto the piranha activated Si substrate at $3000 \mathrm{rpm}$ for $30 \mathrm{~s}$ and annealed in a vacuum oven (Townson \& Mercer EV018) at $170^{\circ} \mathrm{C}$ for $6 \mathrm{~h}$. Nonchemically bound polymer was removed by sonication (Cole-Palmer 8891 sonicator) and rinsing in toluene.
2.4. Thin Film Preparation and Solvent Annealing. PS-bPFS BCP was dissolved in toluene at room temperature to yield a $1.0 \mathrm{wt} . \%$ polymer solution. PS- $b$-PFS thin films were fabricated by spin coating typically at $3000 \mathrm{rpm}$ and a $30 \mathrm{~s}$ from a $1.0 \mathrm{wt} \%$ toluene solution to give a film of approximately $50 \mathrm{~nm}$ thick. The films were deposited on clean substrates with a variety of surface chemistries. As-cast films were solvent annealed in glass jars $(150 \mathrm{~mL})$ under a saturated solvent environment at room temperature $\left(\sim 15^{\circ} \mathrm{C}\right)$ for different periods. Samples were removed from the glass jars after the desired anneal time and allowed to evaporate the trapped solvent at ambient conditions.

\subsection{Characterization}

Contact Angle. Static contact angles $\left(\theta_{a}\right)$ of DI water were measured at ambient temperature using a Data Physics Contact Angle (model: OCA15) goniometer. Contact angles were measured on the opposite edges of at least five drops and averaged. The values were reproducible to within $2^{\circ}$.

Film Thickness. Film thickness was determined by ellipsometry (Plasmos SD2000 Ellipsometer) at a fixed incidence angle of $70^{\circ}$. An average of five readings collected from different locations on a sample surface is reported.

Atomic Force Microscopy (AFM). Atomic force microscope (DME 2452 DualScope Scanner DS AFM) was operated in AC (tapping) mode under ambient conditions using silicon microcantilever probe tips with a force constant of $60,000 \mathrm{~N} \mathrm{~m}^{-1}$ and a scanning force of $0.11 \mathrm{nN}$. Topographic and phase images were recorded simultaneously.

\section{Results and Discussion}

3.1. Solvent Effects on Microphase Separation. Previous investigations into PS- $b$-PFS thin films have focused on the use of thermal annealing as a means of inducing microphase separation in the film $[16,17]$. Another approach used to induce microphase separation in BCP thin films is to adopt a solvent exposure technique [19-23] in which the polymer film is exposed to a solvent saturated environment. This technique produces ordered structures that achieve microphase separation via either a controlled solvent evaporation rate from the film [24] or manipulation of surface energies [25]. The basis of the solvent exposure process is the high residence time of the solvent in the film, which provides adequate mobility to allow the system to obtain the most thermodynamically stable phase separated state [26].

A film that has undergone an optimized microphase separation would form hexagonally close-packed (HCP) cylinders with two possible orientations; perpendicular to the substrate (exhibited by a HCP dot structure at the surface) and parallel to substrate (exhibited by a line/fingerprint structure at the surface). Figure 1 shows an as-cast film of PS- $b$-PFS on Si substrate, which has not been annealed. This film exhibits a random dispersion of PFS domains in the PS matrix, 


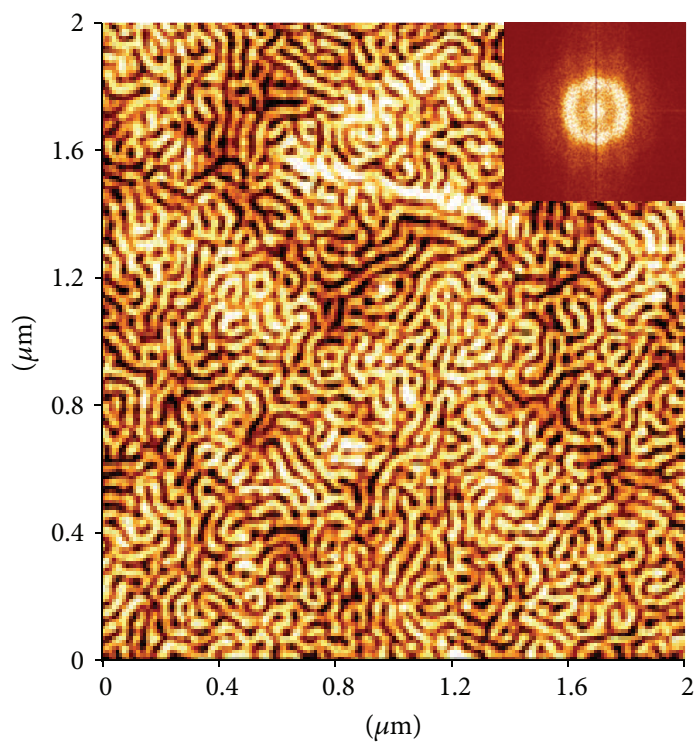

(a)

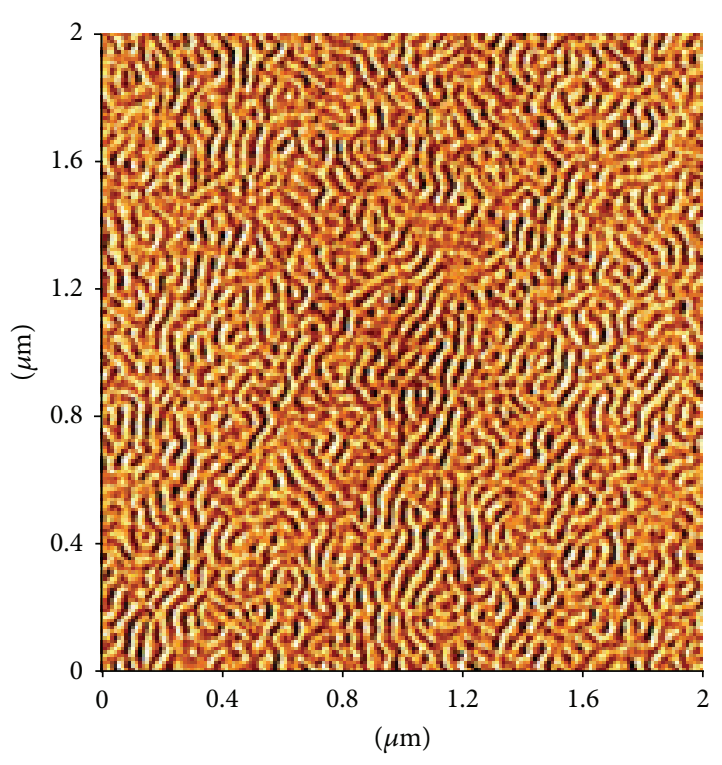

(b)

FIGURE 1: AFM topography (a) and phase (b) images of PS- $b$-PFS thin films on Si substrates prepared from 1.0 wt.\% solution of the polymer in toluene prior to any annealing.

with no clear pattern. This is due to the rapid formation of nonequilibrium domains of PFS, which are formed in the PS matrix during the spin casting process. During the spin casting process there is sufficient solvent present in the film to allow the PFS microdomains to begin to form within the PS matrix. However, before the equilibrium state can be achieved the solvent is evaporated, essentially freezing the morphology in this nonequilibrium state. The corresponding FFT image, inserted in Figure 1(a), displays a broad indistinct bright ring confirming the lack of orientational alignment present. The width/indistinctness of the ring indicates a disordered arrangement with a range of pitch periods, indicating that the random PFS domains formed are of various sizes and that there is a wide orientation distribution of the domains.

Choice of solvent is critical in optimizing the phase separation of the PS- $b$-PFS thin films using the solvent annealing technique, and the thin films on Si substrates were exposed to wide variety of solvent atmospheres, six of which are shown below in Figure 2. All structures observed are typical of a morphology of cylinders orientated parallel, or perpendicular, to the substrate surface. When exposed to a toluene atmosphere for $3 \mathrm{~h}$ the film exhibits the structure shown in Figure 2(a). There is a complete lack of microphase separation across the film. This is as a result of the very high mobility that toluene provides both PS and PFS, allowing the blocks to move beyond microphase separation into macrostructures, for example, as two layer domains. The corresponding FFT, inserted in Figure 2(a), has no structure, confirming that there is no periodicity between the dark and light regions of the AFM image. Exposure to an acetone atmosphere results in increased ordering and length of the PFS, as they begin to achieve the equilibrium structure resulting in the beginnings of a stripe pattern, Figure 2(b). The FFT image, inserted in Figure 2(b), displays a complete ring where the width of the bright line is much narrower compared with the ring obtained for film that was analyzed as-cast, Figure 1, suggesting that the cylinder spacing is becoming more regular. The presence of a complete ring is due to the wide orientation distribution of the domains. When exposed to an atmosphere of hexane, Figure 2(c), there is no improvement in the microphase separation in the film from that observed in an as-cast film. The FFT, inserted Figure 2(c), is also similar to that of the as-cast film, with a broad, indistinct ring. With heptane, Figure 2(d), there is only some minor impovement over an ascast film in the ordering and length of the PFS domains. This is confirmed by the FFT, inserted in Figure 2(d), which shows a much well defined ring, suggesting that the cylinder spacing is becoming more regular. Upon exposure to a cyclohexane environment, the film's morphology has changed, with the majority of PFS cylinders now orienting perpendicular to the substrate instead of parallel, Figure 2(e). The FFT, inserted in Figure 2(e), shows a complete ring, confirming that there is little or no long range hexagonal ordering in the dot pattern, as this would appear on an FFT as 6 distinct dots. However, the ring is well defined, indicating that cylinder spacing is quite uniform. Exposing the film to a THF atmosphere yielded the best results, as can be seen in Figure 2(f). The disordered domains of the as-cast film have developed into a "fingerprint" pattern of PFS cylinders in a PS matrix parallel to the surface. The development of this "fingerprint" pattern is the result of the reduction in the number of grain boundaries, as larger grains are formed, further reducing the overall energy of the system. Rather than isolated grains shrinking, this process involves the collapse of smaller grains into the grain boundaries of larger grains [27]. The FFT image, inserted in Figure 2(f), displays two arcs, which are mirror images of each other and equidistant from the center point. As a ring pattern is no longer present and only arcs remain indicate that the orientation distribution of the domains has decreased, consistent with the observed decrease in grain 


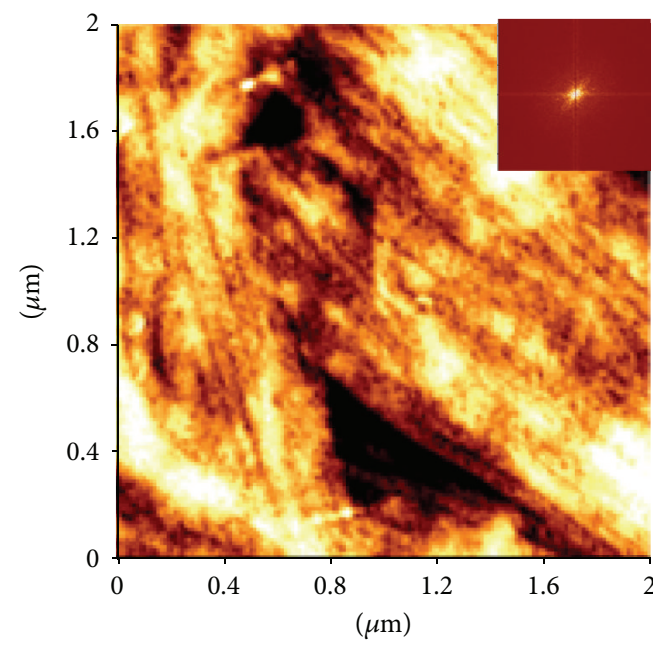

(a)

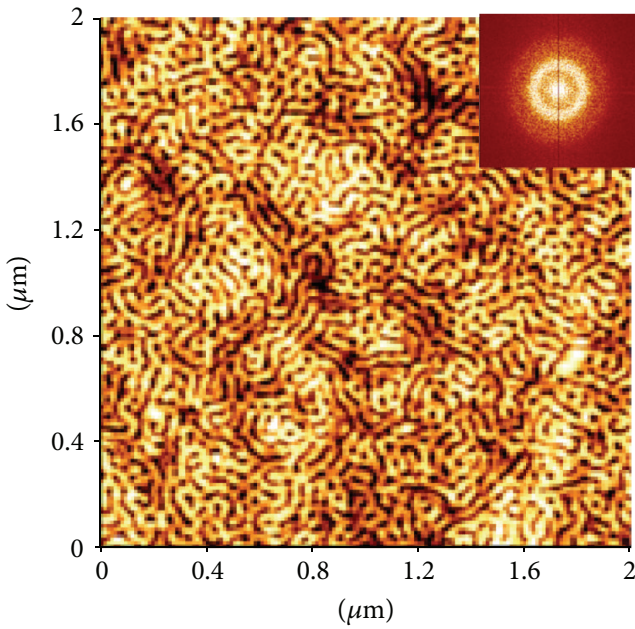

(c)

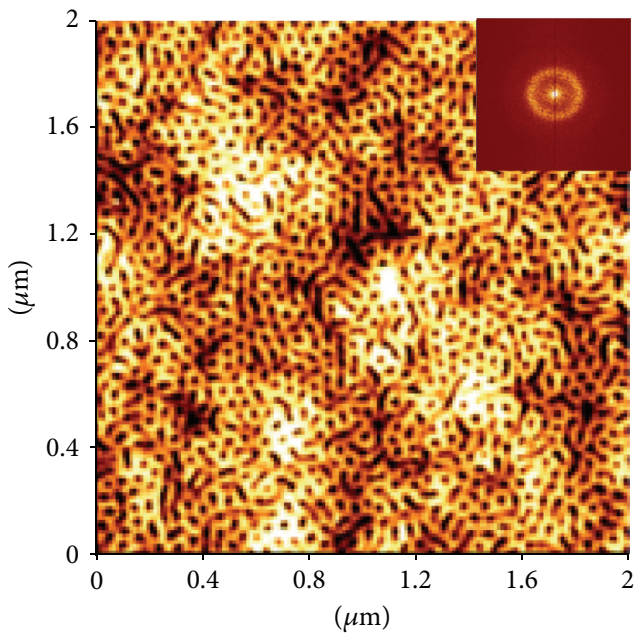

(e)

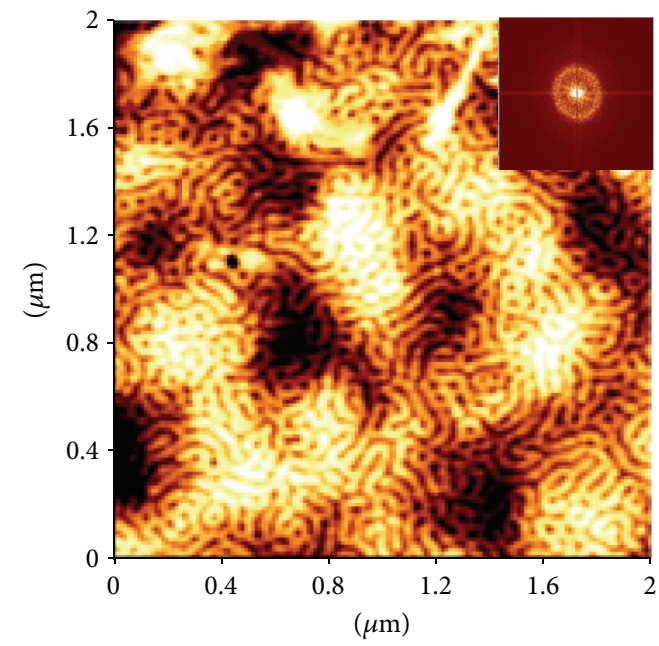

(b)

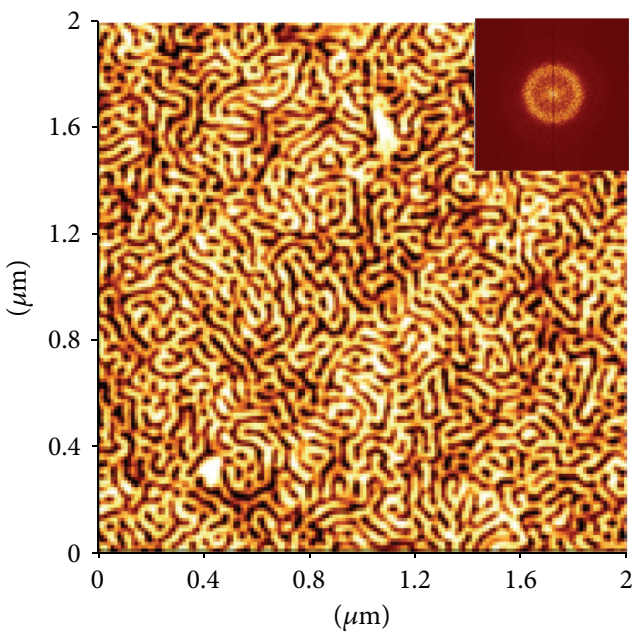

(d)

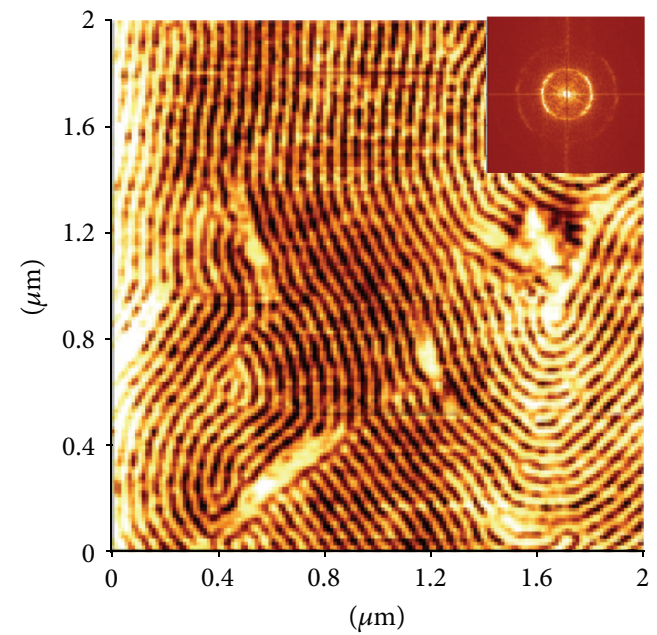

(f)

FIGURE 2: AFM topography images of PS- $b$-PFS thin films on Si substrates prepared from $1.0 \mathrm{wt}$ \% solution of the polymer in toluene after $3 \mathrm{~h}$ exposure to (a) toluene, (b) acetone, (c) hexane, (d) heptane, (e) cyclohexane, and (f) THF atmospheres at room temperature. 
TABLE 1: Hildebrand solubility parameter $\left(\delta_{H}\right)$ of solvents.

\begin{tabular}{lc}
\hline Solvent & $\left(\delta_{H}\right) / \mathrm{MPa}^{1 / 2}$ \\
\hline Acetone & 19.7 \\
Cyclohexane & 16.8 \\
n-Heptane & 15.3 \\
n-Hexane & 14.9 \\
PFS & 18.7 \\
PS & 18.0 \\
THF & 18.5 \\
Toluene & 18.3 \\
\hline
\end{tabular}

boundaries. That the 2 nd harmonic is visible on the FFT further confirms the high level of ordering and uniformity of the feature size.

The last two experiments, cyclohexane and THF solvent anneal, show different results than might have been expected. The structure of a PS- $b$-PFS film, with a PFS volume fraction $\left(f_{\mathrm{PFS}}\right)$ of 0.33 , is expected to be a hexagonal arrangement of PFS cylinders in a PS matrix. A stripe or dot pattern is formed depending on whether the PFS cylinders are orientated parallel or perpendicular to the substrate, respectively. Current theories relating polymer morphology to the Hildebrand solubility parameters $\left(\delta_{H}\right)$ of the solvent during solvent anneals do not provide a sufficient explanation of the structures observed $[28,29]$. Indeed, the formation of a parallel orientation of the PFS $\left(18.7 \mathrm{MPa}^{1 / 2}\right)$ cylinders should be expected for cyclohexane as the Hildebrand solubility parameter of the cyclohexane vapour $\left(16.8 \mathrm{MPa}^{1 / 2}\right)$ is closer to that of PS $\left(18 \mathrm{MPa}^{1 / 2}\right)$ and we would expect a structure that minimizes the exposure of PFS at the polymer/air interface. In the case of THF $\left(18.5 \mathrm{MPa}^{1 / 2}\right)$ which has a Hildebrand solubility parameter between PS and PFS, the anticipated result would be perpendicular orientation of PFS, to maximize the exposure of both PS and PFS at the surface. As can be seen from Figures 2(e) and 2(f), this is not the case; the cyclohexane is exhibiting a perpendicular orientation, while the THF exhibits a parallel orientation. For convenience, the Hildebrand solubility parameter of the solvents used in the study along with PS and PFS is listed in Table 1. We suggest that in this system, with the very similar surface energies of the two constituent polymer blocks $(\sim 40 \mathrm{mN} / \mathrm{m})$ [30], that instead of the usual structure of vertically aligned cylindricalforming films, where only the majority block is present at the film/air interface, Scheme 1(a), that PS- $b$-PFS forms a structure where the PFS cylinders are exposed at the surface, Scheme 1(b). If the cylinders are exposed at the surface, then a vertical alignment would be minimizing PFS exposure (in agreement with cyclohexane result), while a horizontal alignment would be close to a 50/50 exposure of both blocks (in agreement with the THF result).

3.2. Anneal Time Effects on Microphase Separation. Another important factor in the optimization of microphase separation is the time that the film is exposed to the annealing conditions, in this case the solvent atmosphere. Exposure time can affect the microphase separation of a film in several

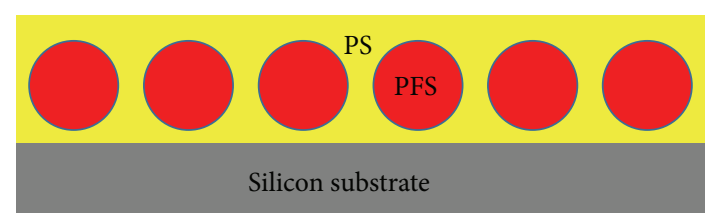

(a)

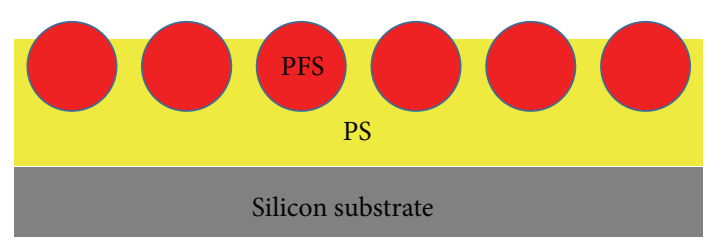

(b)

SCHEME 1: Schematics of the possible structures of PS- $b$-PFS thin films on a substrate, (a) PFS cylinders contained within the PS matrix, and (b) PFS cylinders exposed at the surface.

ways, for example, allowing enough time for the solvent vapour to become saturated in the sealed environment and/or to allow a suitable period for mass transport to occur. A simple example of this can be seen with as-cast films of PS- $b$-PFS. As mentioned previously, an as-cast film exhibits a random dispersion of PFS domains in the PS matrix, with no obvious highly ordered alignment; see Figure 1. This probably occurs because during the spin casting process there is sufficient solvent present to facilitate limited mass transport. However, before the equilibrium state can be achieved the solvent is evaporated, freezing the morphology in this nonequilibrium state. This is a clear demonstration of the importance of the residency time of the solvent vapour within the film.

When exposed to a THF atmosphere for $15 \mathrm{~min}$ the film on Si substrate exhibits the structure shown in Figure 3(a). The film displays no significant increase in the ordering of the PFS cylinders within the PS matrix over the as-cast film, Figure 1. The FFT, inserted in Figure 3(a), is also similar to that of the as-cast film, with a broad, indistinct ring, confirming the lack of lateral order present in the film. We suggest that this lack of ordering in the microphase separation of the film is most likely due to the short annealing time, which prevents the THF vapour from achieving sufficient concentration within the film to alter block mobility or the residency time of the THF within the film is too short to achieve a sufficient mass transport that would increase the level of ordering over that of as-cast film. With the lengthening of the time period to $30 \mathrm{~min}$, there is a significant increase in the size and ordering of the PFS domains, as the film moves through an intermediate structure towards equilibrium, Figure 3(b). While the majority of the PFS domains have the expected parallel orientation, some domains perpendicular to the substrate can be identified on the AFM image. The fact that the domains are sufficiently mobile to be able to reorientate from parallel to perpendicular is a clear indication that there is adequate solvent within the film after $30 \mathrm{~min}$. There are, however, a high number of energetically unfavourable grain 


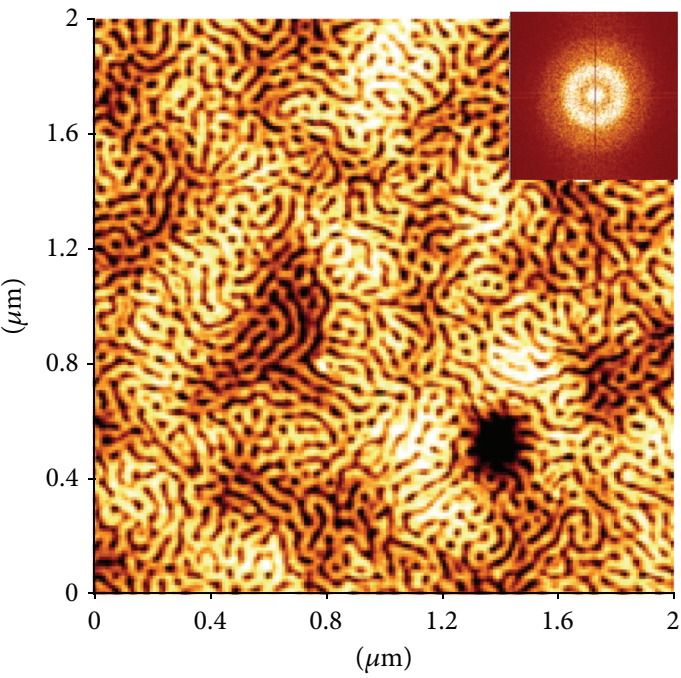

(a)

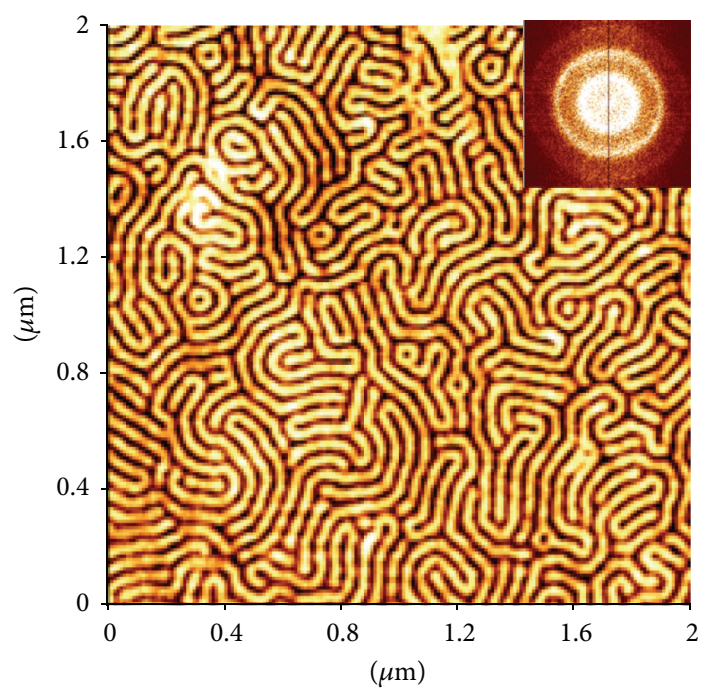

(c)

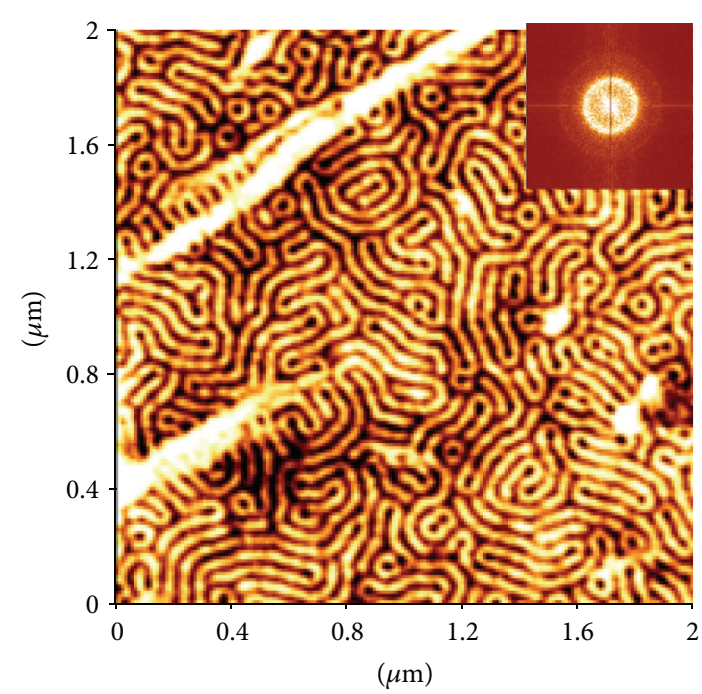

(b)

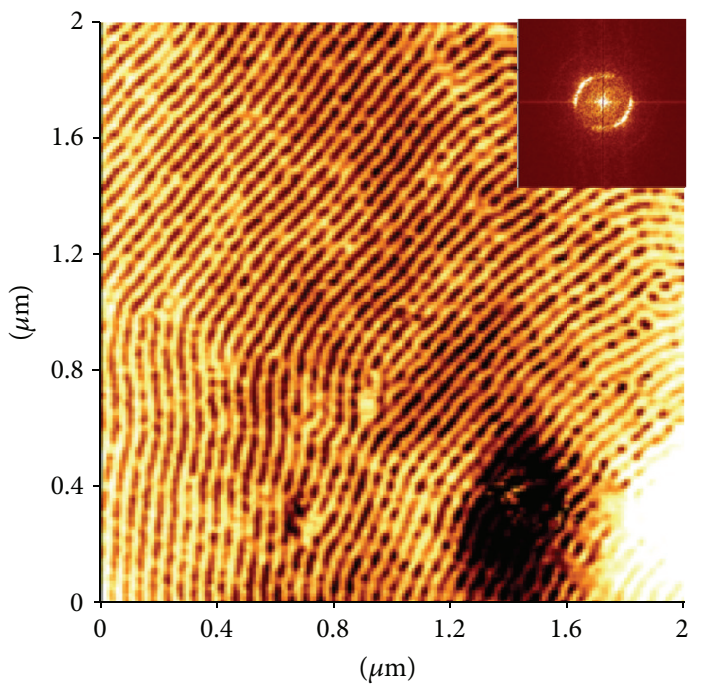

(d)

FIGURE 3: AFM topography images of PS- $b$-PFS thin films on Si substrates prepared from $1.0 \mathrm{wt} . \%$ solution of the polymer in toluene after exposure to a THF for (a) $15 \mathrm{~min}$, (b) $30 \mathrm{~min}$, (c) $45 \mathrm{~min}$, and (d) $1 \mathrm{~h}$ at room temperature.

boundaries present. Within each grain the PFS cylinders are parallel to one another; however, due to the mismatch of hexagonal lattice of the cylinders grain boundaries are formed [28]. The high number of "grains" present is evidence of a random nucleation mechanism of cylinder formation, where a large number of nucleation sites occur randomly throughout the system. The corresponding FFT, inserted in Figure 3(b), shows a better defined ring structure indicating a more regular domain size, along with the presence of the 2nd and 3rd harmonic confirming the increased ordering of the film.

After 45 min solvent exposure, there is a further improvement in the ordering of the PFS domains, Figure 3(c). The increase in ordering is the result of the reduction in the number of grain boundaries as larger grains are formed, further reducing the overall energy of the system. This process involves the collapse of smaller grains into the grain boundaries of larger grains [31]. There are still a few isolated perpendicular domains that indicate that the film is still in transition to the equilibrium structure. At $1 \mathrm{~h}$, all of the submicron grains have collapsed into the larger ones and ordering now extends into the 2 to $5 \mu \mathrm{m}$ range. The FFT image, inserted in Figure 3(d), displays two arcs, which are mirror images of each other and equidistant from the center point. As a ring pattern is no longer present and only arcs remain, this indicates that the orientation distribution of the domains has decreased, consistent with the observed decrease in grain boundaries. The arcs also have a very narrow band confirming a highly regular domain size distribution. Further annealing does not result in a noticeable improvement in the overall order of the system and instead leads to an increase in the amount of dewetting the film undergoes on the substrate. 
TABLE 2: Static water contact angle within experimental error $( \pm 2)$ for PS, PFS, and substrates with various chemistries.

\begin{tabular}{lcccccccc}
\hline Surface type & $\mathrm{PS} / \mathrm{Si}$ & $\mathrm{PFS} / \mathrm{Si}$ & $\mathrm{Si}$ & $\mathrm{Si}_{3} \mathrm{~N}_{4}$ & $\mathrm{SiO}_{x} \mathrm{~N}_{y}$ & $\mathrm{Ge}$ & $\mathrm{W}$ & $\mathrm{Graphene}$ \\
\hline Contact angle, $^{\circ}$ & 112.0 & 109.5 & 32.0 & 46.6 & 46.3 & 91.4 & 67.3 & 86.0 \\
\hline
\end{tabular}

For the reasons stated, solvent annealing of PS- $b$-PFS thin films at room temperature for $1 \mathrm{~h}$ is determined to be the optimum annealing conditions.

\subsection{Structural Stability during Microphase Separation.} Another important consideration in the optimization of a BCP system is the interfacial interaction between the polymer and the substrate [14, 21, 26, 32, 33]. Extensive research has shown that the interactions of the BCP with the substrate can have a significant effect on the microphase separation of the system and can also lead to complex morphologies on the micron scale [34]. BCP systems which have achieved mobility, either through solvent or thermal annealing to effect microphase separation, will display changes in the film structure that occur to minimise surface energies and maximise bonding interactions with the surface. These interactions between the BCP and the substrate are predominately observed in a number of ways; preferential wetting layers by segregation of the preferred block to the substrate or surface; irregular films caused by dewetting of the film and orientational effects and stability of microphase separated patterns.

These types of effects are illustrated in the schematics of Scheme 2 (assuming that the system is lamellar and that substrate surface effects are dominant and surface interface effects can be ignored for simplicity). In Schemes 2(a) and 2(b) two different orientations of the same structure can be seen. In Scheme 2(a) a vertical orientation of the lamellae occurs if both blocks are equally favoured at the substrate surface, whilst in Scheme 2(b) a horizontal orientation occurs as of one blocks is preferentially favoured. This also holds true for a hexagonal cylinder structure, a substrate surface that interacts equally with both blocks will favour vertical orientation of cylinders with respect to the surface, whilst favourable interactions of one block with the surface and substrate interfaces can lead to the formation of wetting layers which will tend to favour a horizontal orientation to the surface. In general, the block that has the lowest surface energy will reside at the surface. Interactions of the BCP with the substrate can also lead to complex morphologies on the micron scale. If a surface is hydrophobic and both blocks in a BCP are sufficiently hydrophilic there will be a tendency to form droplets rather than commensurate films as illustrated in Scheme 2(c) where hemispheres of phase separated polymers are formed [35].

With the importance of substrate chemistry in effecting the morphology of a BCP thin film, investigations into the effect of various substrates on the microphase separation of PS- $b$-PFS thin films were undertaken. Contact angle measurements of PS and PFS homopolymers and substrates of differing surface chemistry were undertaken and the results are compiled in Table 2 . Contact angle measurements from these systems can serve as an indicator of

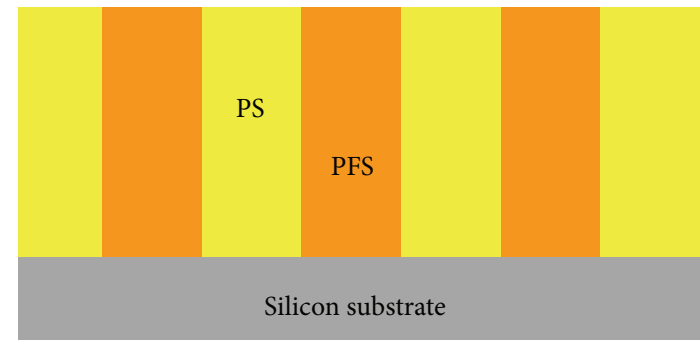

(a)

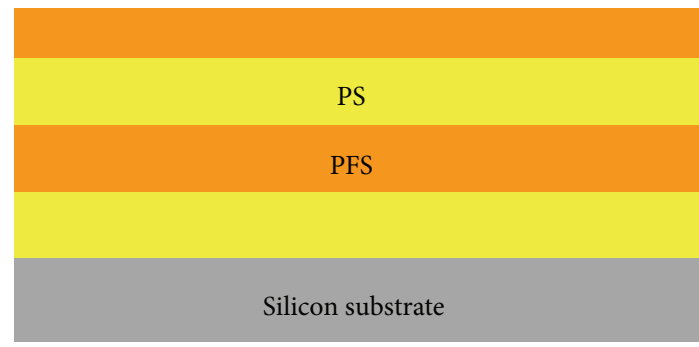

(b)

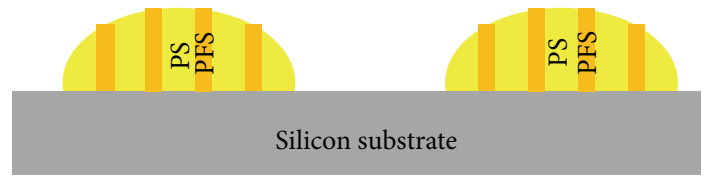

(c)

Scheme 2: Schematic of the effect the polymer/substrate interaction can have on film morphology: (a) both blocks have an affinity for the surface, (b) one block preferentially wets the surface, and (c) both blocks are repulsed by the surface.

the hydrophobicity/hydrophilicity of the substrate. The lower the angle the more hydrophilic the surface (e.g., Si), while the higher the angle the more hydrophobic (e.g., Ge). In a simple case of "like to like", a hydrophobic block will preferentially wet a hydrophobic substrate, while a hydrophilic block will preferentially wet a hydrophilic substrate. But as can be seen from Table 1, PS and PFS are almost identically hydrophobic $\left(112^{\circ}\right.$ and $109^{\circ}$, resp.). With two blocks with such similar surface energies it is unlikely that a change in the substrate chemistry would have a significant effect on their microphase separation.

This is confirmed by the results shown in Figure 4. As can be seen from the images, substrates with varying hydrophobicity/hydrophlicity have no significant effect on the formation of the expected "fingerprint" structure across the substrates. The same level of ordering and feature size that was present on the most hydrophilic of the substrates, $\mathrm{Si}$, is also present on the most hydrophobic graphene, Figure 4(d). These results confirm that the similarities in surface energy 


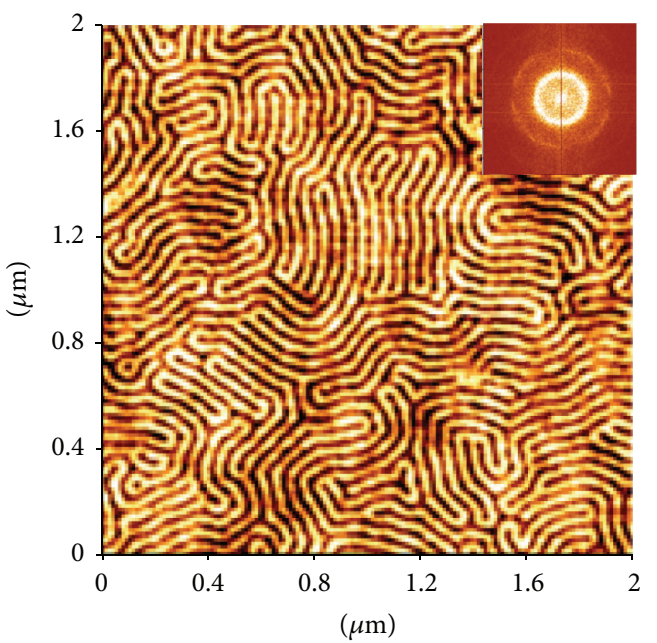

(a)

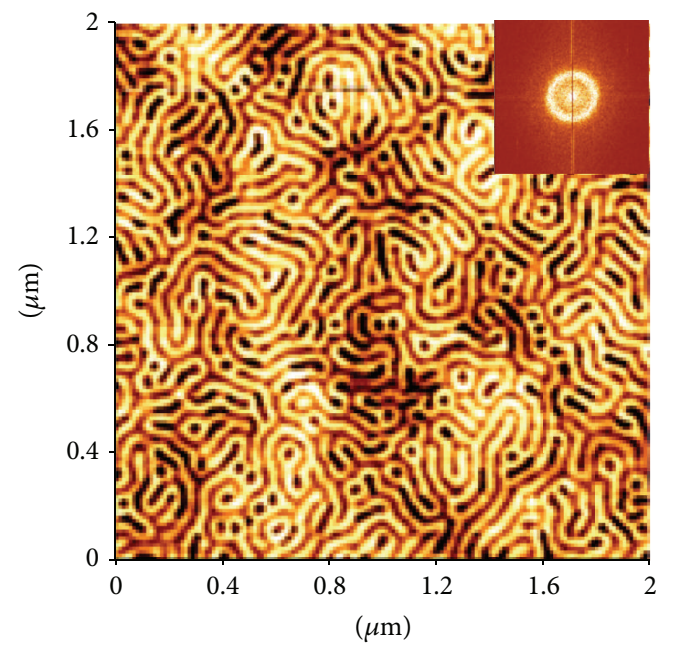

(c)

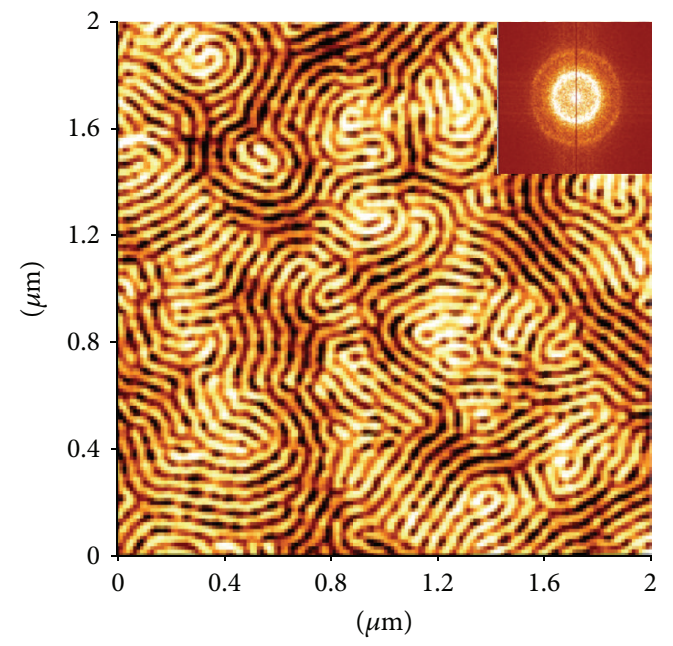

(e)

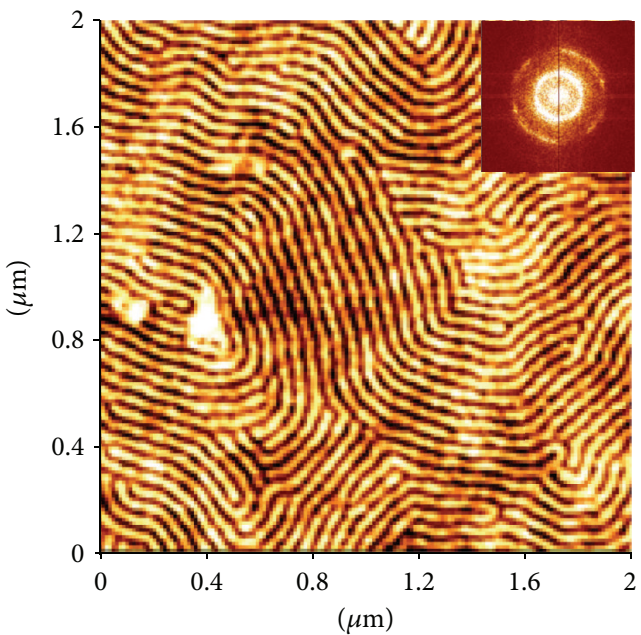

(b)

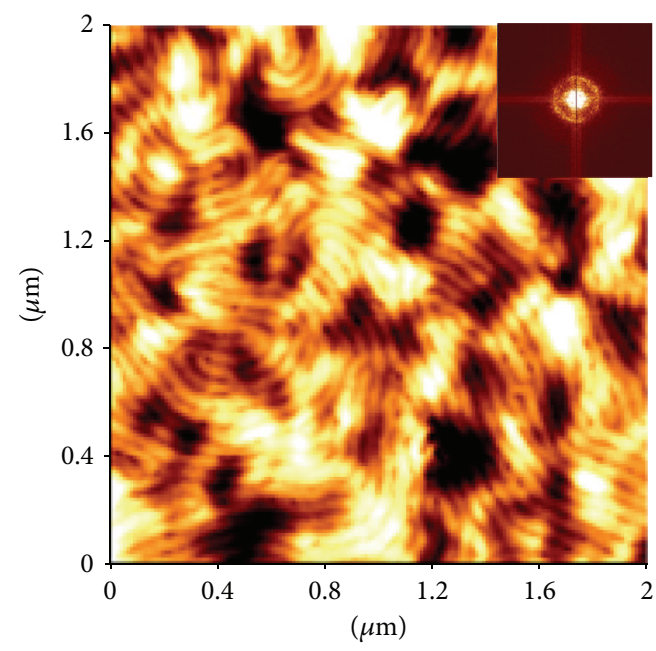

(d)

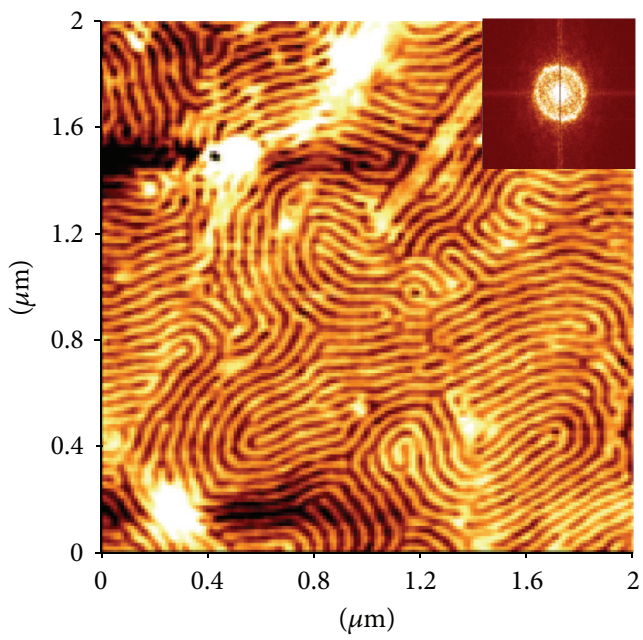

(f)

FIGURE 4: AFM topography images of PS- $b$-PFS thin films after exposure to THF for $1 \mathrm{~h}$ at room temperature prepared from a $1.0 \mathrm{wt} . \%$ solution of the polymer on substrates of (a) $\mathrm{Si}_{3} \mathrm{~N}_{4}$, (b) $\mathrm{SiO}_{x} \mathrm{~N}_{y}$ (c) Ge, (d) W, (e) polymer brush modified $\mathrm{Si}$, and (f) graphene. 
of PS and PFS mean that there is little or no substrate effect on the microphase separation of PS- $b$-PFS.

\section{Conclusions}

Microphase separation, resulting in the formation of highly ordered nanostructures, can be readily achieved in PS- $b$-PFS BCP thin films via solvent annealing. During this process solvent is incorporated into the polymer film providing mobility to the polymer chains effectively lowering the glass transition temperature of the system. Solvent annealing in THF results in ordered structures of PFS cylinders orientated parallel to the substrate in the PS matrix, while a cyclohexane environment resulted in a perpendicular orientation. Given current theories regarding the connection between the Hildebrand solubility parameters $\left(\delta_{H}\right)$ of the solvent and the polymer blocks and the structural orientation of a film, these results lead to the proposal of new structure for cylindrical PS- $b$-PFS thin films, where the PFS cylinders are exposed above the PS matrix. With both PS and PFS exhibiting similar surface energies, the chemistry of the substrate at the film interface has little to no effect on the system. This gives PS$b$-PFS thin films a very high level of structural stability across a wide variety of substrate chemistries.

\section{Conflict of Interests}

The authors declare that there is no conflict of interests regarding the publication of this paper.

\section{Acknowledgments}

Financial support for this work is provided by the EU FP7 NMP project, LAMAND (Grant no. 245565) project, and the Science Foundation Ireland (Grant no. 09/IN.1/602) and is gratefully acknowledged. The contents of this work are the sole responsibility of the authors. The authors also thank Intel, Ireland, for substrates.

\section{References}

[1] D. Borah, M. T. Shaw, S. Rasappa et al., "Plasma etch technologies for the development of ultra-small feature size transistor devices," Journal of Physics D: Applied Physics, vol. 44, no. 17, Article ID 174012, 2011.

[2] Y. N. Xia and G. M. Whitesides, "Soft lithography," Annual Review of Materials Science, vol. 28, no. 1, pp. 153-184, 1998.

[3] C. J. Hawker and T. P. Russell, "Block copolymer lithography: merging 'bottom-up' with 'top-down' processes," MRS Bulletin, vol. 30, no. 12, pp. 952-966, 2005.

[4] M. Park, C. Harrison, P. M. Chaikin, R. A. Register, and D. H. Adamson, "Block copolymer lithography: periodic arrays of $10^{11}$ holes in 1 square centimeter," Science, vol. 276, no. 5317, pp. 1401-1404, 1997.

[5] T. Xu, J. Stevens, J. Villa et al., "Block copolymer surface reconstruction: a reversible route to nanoporous films," Advanced Functional Materials, vol. 13, no. 9, pp. 698-702, 2003.

[6] A. Nunns, J. Gwyther, and I. Manners, "Inorganic block copolymer lithography," Polymer, vol. 54, no. 4, pp. 1269-1284, 2013.
[7] J. Bodycomb, Y. Funaki, K. Kimishima, and T. Hashimoto, "Single-grain lamellar microdomain from a diblock copolymer," Macromolecules, vol. 32, no. 6, pp. 2075-2077, 1999.

[8] G. Kim and M. Libera, "Morphological development in solvent-cast polystyrene-polybutadiene-polystyrene (SBS) triblock copolymer thin films," Macromolecules, vol. 31, no. 8, pp. 2569-2577, 1998.

[9] S. H. Kim, M. J. Misner, T. Xu, M. Kimura, and T. P. Russell, "Highly oriented and ordered arrays from block copolymers via solvent evaporation," Advanced Materials, vol. 16, no. 3, pp. 226231, 2004.

[10] D. A. Rider, K. Liu, J.-C. Eloi et al., "Nanostructured magnetic thin films from organometallic block copolymers: pyrolysis of self-assembled polystyrene-block-poly(ferrocenylethylmethylsilane)," ACS Nano, vol. 2, no. 2, pp. 263-270, 2008.

[11] J. G. Son, J. Gwyther, J.-B. Chang, K. K. Berggren, I. Manners, and C. A. Ross, "Highly ordered square arrays from a templated ABC triblock terpolymer," Nano Letters, vol. 11, no. 7, pp. 2849$2855,2011$.

[12] T. Thurn-Albrecht, J. Derouchey, T. P. Russell, and H. M. Jaeger, "Overcoming interfacial interactions with electric fields," Macromolecules, vol. 33, no. 9, pp. 3250-3253, 2000.

[13] S. O. Kim, H. H. Solak, M. P. Stoykovich, N. J. Ferrier, J. J. de Pablo, and P. F. Nealey, "Epitaxial self-assembly of block copolymers on lithographically defined nanopatterned substrates," Nature, vol. 424, no. 6947, pp. 411-414, 2003.

[14] D. Borah, R. Senthamaraikannan, S. Rasappa, B. Kosmala, J. D. Holmes, and M. A. Morris, "Swift nanopattern formation of PS- $b$-PMMA and PS- $b$-PDMS block copolymer films using a microwave assisted technique," ACS Nano, vol. 7, no. 8, pp. 6583-6596, 2013.

[15] E. Drockenmuller, L. Y. T. Li, D. Y. Ryu et al., "Covalent stabilization of nanostructures: robust block copolymer templates from novel thermoreactive systems," Journal of Polymer Science Part A: Polymer Chemistry, vol. 43, no. 5, pp. 1028-1037, 2005.

[16] R. G. H. Lammertink, M. A. Hempenius, J. E. van den Enk, V. Z.-H. Chan, E. L. Thomas, and G. J. Vancso, "Nanostructured thin films of organic-organometallic block copolymers: onestep lithography with poly(ferrocenylsilanes) by reactive ion etching," Advanced Materials, vol. 12, no. 2, pp. 98-103, 2000.

[17] L. Cao, J. A. Massey, M. A. Winnik et al., "Reactive ion etching of cylindrical polyferrocenylsilane block copolymer micelles: fabrication of ceramic nanolines on semiconducting substrates," Advanced Functional Materials, vol. 13, no. 4, pp. 271-276, 2003.

[18] M. Ramanathan and S. B. Darling, “Thickness dependent hierarchical meso/nano scale morphologies of a metal-containing block copolymer thin film induced by hybrid annealing and their pattern transfer abilities," Soft Matter, vol. 5, no. 23, pp. 4665-4671, 2009.

[19] X. Gu, I. Gunkel, A. Hexemer, W. Gu, and T. P. Russell, "An in situ grazing incidence X-ray scattering study of block copolymer thin films during solvent vapor annealing," Advanced Materials, vol. 26, no. 2, pp. 273-281, 2014.

[20] C. Sinturel, M. Vayer, M. Morris, and M. A. Hillmyer, "Solvent vapor annealing of block polymer thin films," Macromolecules, vol. 46, no. 14, pp. 5399-5415, 2013.

[21] D. Borah, M. Ozmen, S. Rasappa, M. T. Shaw, J. D. Holmes, and M. A. Morris, "Molecularly functionalized silicon substrates for orientation control of the microphase separation of PS- $b$ PMMA and PS- $b$-PDMS block copolymer systems," Langmuir, vol. 29, no. 9, pp. 2809-2820, 2013. 
[22] A. Sperschneider, F. Schacher, M. Gawenda et al., "Towards nanoporous membranes based on ABC triblock terpolymers," Small, vol. 3, no. 6, pp. 1056-1063, 2007.

[23] M. Lazzari, G. Liu, and S. Lecommandoux, Eds., Block Copolymers in Nanoscience, Wiley-VCH, Weinheim, Germany, 1st edition, 2006.

[24] Z. Q. Lin, D. H. Kim, X. D. Wu et al., "A rapid route to arrays of nanostructures in thin films," Advanced Materials, vol. 14, no. 19, pp. 1373-1376, 2002.

[25] S. Kim, R. M. Briber, A. Karim, R. L. Jones, and H.-C. Kim, "Environment-controlled spin coating to rapidly orient microdomains in thin block copolymer films," Macromolecules, vol. 40, no. 12, pp. 4102-4105, 2007.

[26] D. Borah, S. Rasappa, R. Senthamaraikannan et al., "Orientation and alignment control of microphase-separated PS- $b$-PDMS substrate patterns via polymer brush chemistry," ACS Applied Materials and Interfaces, vol. 5, no. 1, pp. 88-97, 2013.

[27] C. Harrison, D. E. Angelescu, M. Trawick et al., "Pattern coarsening in a 2D hexagonal system," Europhysics Letters, vol. 67, no. 5, pp. 800-806, 2004.

[28] T. G. Fitzgerald, R. A. Farrell, S. O'driscoll, C. T. O'mahony, J. D. Holmes, and M. A. Morrisy, "Orientation and translational control of PS-b-PEO/PS thin films via solvent annealing and graphoepitaxy techniques," e-Journal of Surface Science and Nanotechnology, vol. 7, pp. 471-475, 2009.

[29] S. M. O’Driscoll, C. T. O’Mahony, R. A. Farrell, T. G. Fitzgerald, J. D. Holmes, and M. A. Morris, "Toroid formation in polystyrene-block-poly(4-vinyl pyridine) diblock copolymers: combined substrate and solvent control," Chemical Physics Letters, vol. 476, no. 1-3, pp. 65-68, 2009.

[30] P. F. Green, T. M. Christensen, T. P. Russell, and R. Jerome, "Surface interaction in solvent-cast polystyrene/poly(methyl methacrylate) diblock copolymers," Macromolecules, vol. 22, no. 5, pp. 2189-2194, 1989.

[31] H. Jinnai, K. Yasuda, and T. Nishi, “Three-dimensional observations of grain boundary morphologies in a cylinder-forming block copolymer," Macromolecular Symposia, vol. 245-246, pp. 170-174, 2006.

[32] T. P. Russell, G. Coulon, V. R. Deline, and D. C. Miller, "Characteristics of the surface-induced orientation for symmetric diblock PS/PMMA copolymers," Macromolecules, vol. 22, no. 12, pp. 4600-4606, 1989.

[33] I. In, Y. H. La, S. M. Park, P. F. Nealey, and P. Gopalan, "Side-chain-grafted random copolymer brushes as neutral surfaces for controlling the orientation of block copolymer microdomains in thin films," Langmuir, vol. 22, no. 18, pp. 78557860, 2006.

[34] A. Knoll, K. S. Lyakhova, A. Horvat et al., "Direct imaging and mesoscale modelling of phase transitions in a nanostructured fluid," Nature Materials, vol. 3, no. 12, pp. 886-890, 2004.

[35] Y.-S. Seo, E. Kim, S. Y. Kwon, H. Jing, and K. Shin, "AFM study of phase-separated morphology in immiscible blend thin films," Ultramicroscopy, vol. 108, no. 10, pp. 1186-1190, 2008. 

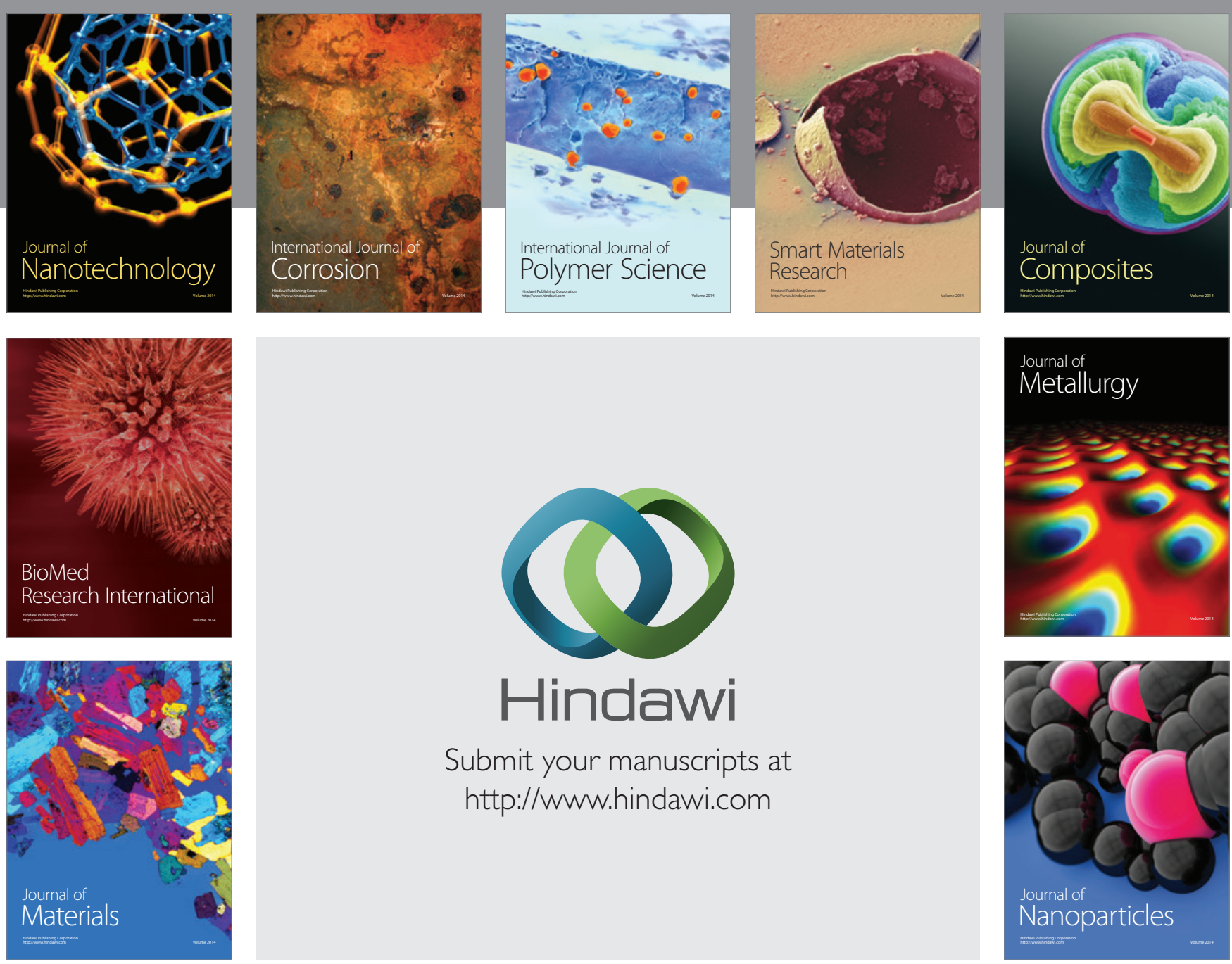

Submit your manuscripts at http://www.hindawi.com
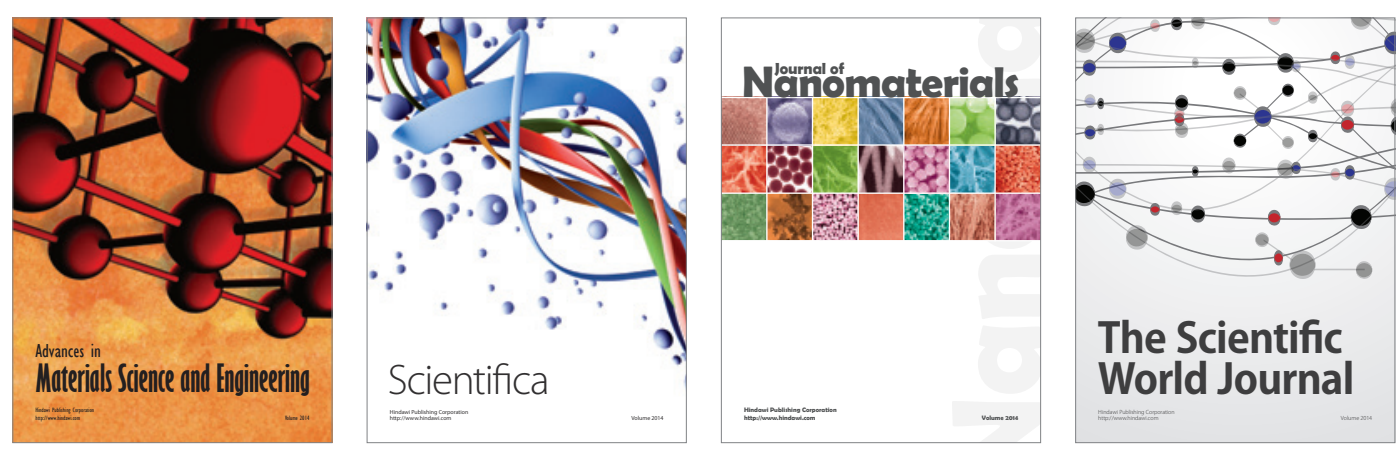

\section{The Scientific World Journal}
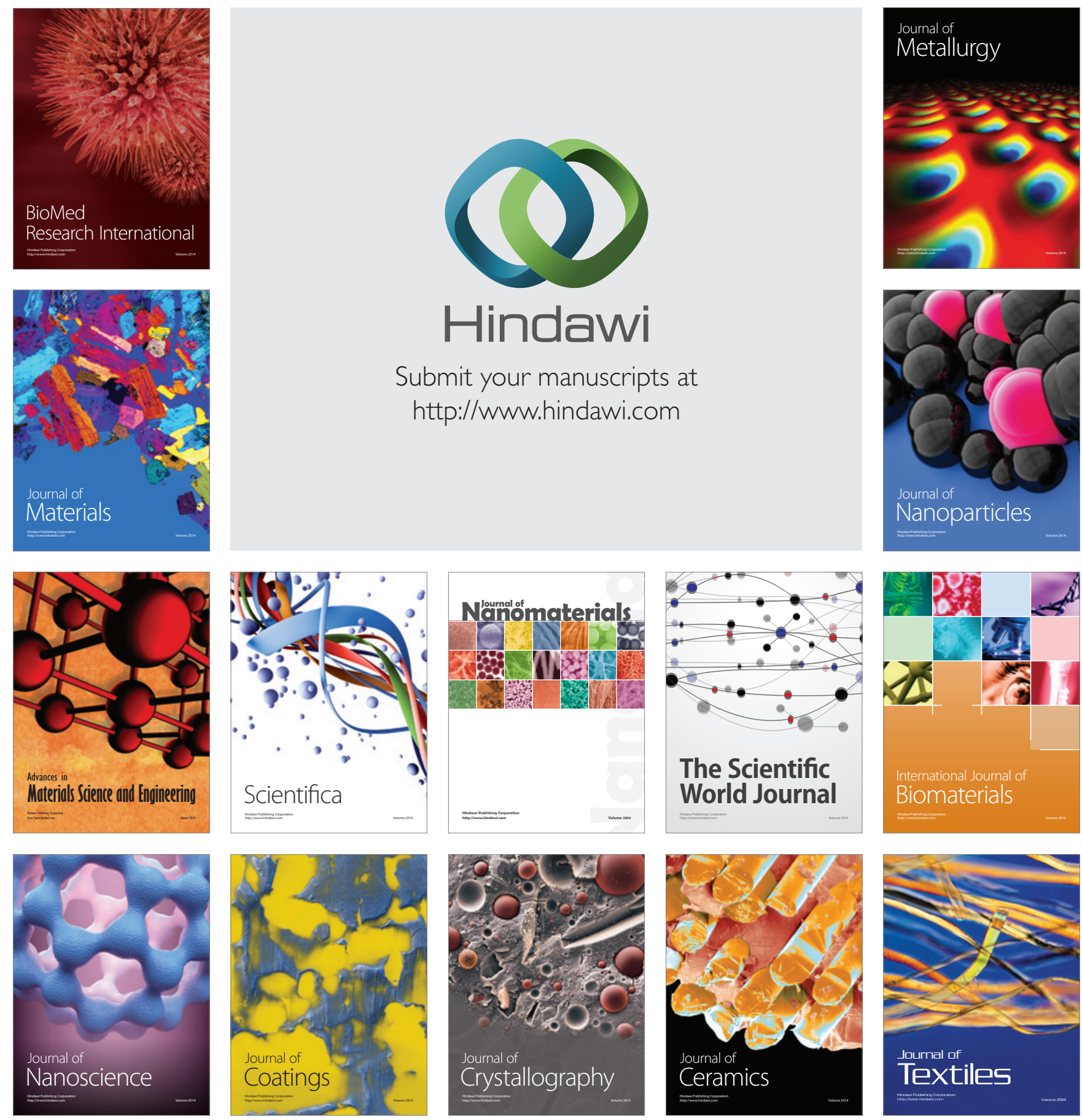